\title{
Il ruolo del nefrologo nella diagnosi e nel follow-up del paziente affetto da malattia di Fabry: I'importanza della gestione multidisciplinare
}

\author{
Leonardo Caroti, Lino Calogero Cirami, Egrina Dervishi, Enrico Eugenio Minetti \\ Nefrologia, Dialisi e Medicina del Trapianto, AOUC Azienda Ospedaliero-Universitaria Careggi, Firenze
}

\begin{abstract}
The role of the nephrologist in the diagnosis and follow-up of patients with Fabry's disease: the importance of a multidisciplinary approach

Fabry's disease (FD) is an X-linked lysosomal storage disorder caused by deficient activity of alpha-galactosidase A, resulting in the accumulation of the glycosphingolipid globotriaosylceramide (Gb3). FD is characterized by the involvement of several systems including the renal, neurological, cardiovascular, cochleovestibular, ocular, gastrointestinal and cutaneous systems. Hemizygous male individuals are primarily affected. Heterozygous females may display moderate to severe disease, which is likely related to the pattern of X-chromosome inactivation. There is a wide spectrum of FD severity. In the classic form of the disease, the clinical manifestations start in childhood and worsen over time according to the glycosphingolipid deposition. In a small percentage of patients with residual enzyme activity the onset of symptoms is late and affects mainly the heart (late-onset variants). Substrate storage affects the kidney, leading to nephropathy as one of the major complications of FD; this may occur in both sexes. These patients develop proteinuria and progress rapidly to chronic renal failure leading to end-stage renal disease. Enzyme replacement therapy and chaperone therapy have been demonstrated to improve the course of the disease, especially when started early. Since the diagnosis of FD is difficult to make, these elements put the nephrologist in a crucial position to diagnose the disease and identify patients who need specific therapy. A multidisciplinary approach is vital in the management of the disease. We describe the role of a multidisciplinary team in the diagnosis, treatment and follow-up of Fabry patients.
\end{abstract}

Keywords: Fabry disease, Renal involvement, Metabolic disorders

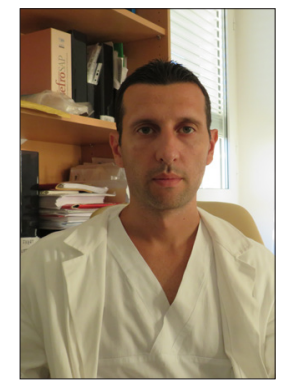

Leonardo Caroti

\section{Introduzione}

La malattia di Fabry rappresenta una lipidosi primaria (1) che determina un accumulo lisosomiale di globotriaosylceramide (Gb3) in vari organi e apparati (rene, cuore, sistema nervoso, cute, apparato oculare, acustico e gastroenterico). La malattia è causata dal deficit dell'enzima alfagalattosidasi $A$ il cui gene è localizzato sul cromosoma $\mathrm{X}$ e, di conse-

Accepted: April 21, 2017

Published online: July 20, 2017

Indirizzo per la corrispondenza:

Dr. Leonardo Caroti

Nefrologia e Dialisi

AOU Careggi

Largo Brambilla, 3

50134 Firenze

carotile@aou-careggi.toscana.it

guenza, il meccanismo di trasmissione risulta di tipo X-linked. In letteratura viene riportata una incidenza pari a 1 caso ogni 40.000/117.000 abitanti (2). La malattia viene classicamente distinta in un fenotipo "classico" (attività enzimatica assente) caratterizzato da un esordio precoce già nella prima infanzia con acroparestesie dolorose e in un fenotipo "late onset" (attività enzimatica $>1 \%$ ) in cui le manifestazioni cliniche sono prevalentemente cardiache ed esordiscono dopo i 30 anni (3). La malattia è in genere più severa nei soggetti di sesso maschile con fenotipo classico; in questi pazienti l'esordio precoce con acroparestesie febbrili può essere seguito dalla comparsa di angiocheratomi cutanei e, nella seconda-terza decade di vita, dall'interessamento renale, cardiaco e del sistema nervoso centrale. Nei soggetti di sesso femminile è possibile osservare un'ampia variabilità di quadri clinici di esordio: dalla forma classica severa con assenza di attività enzimatica, fino a forme con decorso completamente asintomatico e attività enzimatica nella norma. Tale variabilità nell'espressione clinica nei soggetti di sesso femminile è dovuta al fenomeno della lyonizzazione, fenomeno che spiega anche come la malattia in genere sia meno severa e compaia più tardivamente rispetto ai soggetti di sesso maschile (4). Tra i sintomi precoci della malattia di 
TABELLA I - Le manifestazioni precoci e tardive più comuni della malattia di Fabry

\begin{tabular}{|c|c|c|}
\hline Apparato & Sintomi precoci & Sintomi tardivi \\
\hline Sistema nervoso & $\begin{array}{l}\text { Acroparestesie } \\
\text { Ipoacusia } \\
\text { Tinnito } \\
\text { Intolleranza al caldo }\end{array}$ & Ictus \\
\hline Cute & Angiocheratoma, ipoidrosi & \\
\hline Occhio & Opacità corneali & \\
\hline Cuore & $\begin{array}{l}\text { Aritmie } \\
\text { PR corto } \\
\text { Insufficienza valvolare }\end{array}$ & $\begin{array}{l}\text { Disfunzione cardiaca } \\
\text { Cardiopatia ischemica } \\
\text { Difetti di conduzione AV }\end{array}$ \\
\hline
\end{tabular}

$\mathrm{ESRD}=$ end stage renal disease, $\mathrm{PR}=$ intervallo tra onda $\mathrm{P}$ e onda $\mathrm{R} ; \mathrm{AV}=$ atrioventricolare.

Fabry (Tab. I) vengono anche riportati, oltre alle acroparestesie, i disturbi gastrointestinali come i dolori addominali e la diarrea, la cornea verticillata, l'ipo-anidrosi il tinnito, e l'intolleranza al caldo e al freddo (segni di un interessamento del sistema nervoso periferico) e la microalbuminuria. Tra le manifestazioni cliniche successive della malattia occorre ricordare l'interessamento cardiovascolare (cardiomiopatia ipertrofica, aritmie, disfunzione diastolica, valvulopatie, angina), renale (proteinuria, progressione della malattia renale cronica fino all'end stage renal disease-ESRD) e del sistema nervoso centrale (ictus ischemico o emorragico, dolico-ectasia dell'arteria basilare).

Dal punto di vista genetico la malattia presenta caratteristiche estremamente variabili; sono state descritte mutazioni puntiformi missenso, non senso, piccole delezioni, inserzioni e larghe delezioni. La variabilità nell'espressione della malattia è legata anche alla presenza di mutazioni non causative e a varianti atipiche (per esempio, N215S, associata alla cardiomiopatia ipertrofica). L'accumulo progressivo di Gb-3, che può iniziare già in epoca fetale, è associato allo sviluppo di depositi intracellulari a livello lisosomiale che provocano la comparsa di un danno ischemico microvascolare in vari organi e apparati. Le mutazioni associate ad attività enzimatica residua di scarsa entità sono anche quelle in cui si possono osservare i livelli ematici più elevati di globotriaosylsphingosine (lyso-Gb3);

La diagnosi di malattia di Fabry nei soggetti di sesso maschile si avvale della valutazione dell'attività enzimatica dell'enzima alfa-GAL A nei leucociti a cui seguirà, in caso di riscontro di attività enzimatica ridotta, il sequenziamento del gene GLA che dovrà identificare la presenza di una mutazione già descritta in letteratura oppure una variante ignota o un polimorfismo; data la complessità delle mutazioni genetiche considerate e la necessità di allestire un albero genealogico per le famiglie interessate dalla patologia, la consulenza genetica rappresenta un elemento cruciale nella fase diagnostica della malattia. L'analisi enzimatica eseguita su DBS (dried blood spot), dovrebbe essere comunque confermata da procedure standard di laboratorio (5). Nei soggetti di sesso femminile, in caso di sospetto clinico di malattia, è necessario procedere direttamente al sequenziamento del gene GLA per raggiungere la diagnosi della patologia (Fig. 1). In tutti i pazienti affetti da malattia di Fabry la diagnosi dovrebbe avvalersi anche del dosaggio di biomarkers (per esempio, lysoGb3) che nella recente letteratura si stanno dimostrando come un elemento utile per esaminare la progressione e la severità della malattia; inoltre possono facilitare la valutazione della risposta alla terapia enzimatica sostitutiva (ERT) (6).

Dal 2001 è disponibile una terapia enzimatica sostitutiva a base di agalsidasi. Attualmente sono disponibili sul mercato due formulazioni di tale terapia enzimatica rappresentate da agalsidasi alfa (prodotta usando colture di fibroblasti umani e registrata a una dose di 0,2 $\mathrm{mg} / \mathrm{kg}$ ogni due settimane) e agalsidasi beta (prodotta da cellule di criceto e registrata a una dose di $1 \mathrm{mg} / \mathrm{kg}$ ogni due settimane). Entrambe le terapie si sono dimostrate sicure ed efficaci e in grado di rallentare il declino della funzione renale, rallentare o stabilizzare la progressione della cardiomiopatia e ritardare la comparsa 


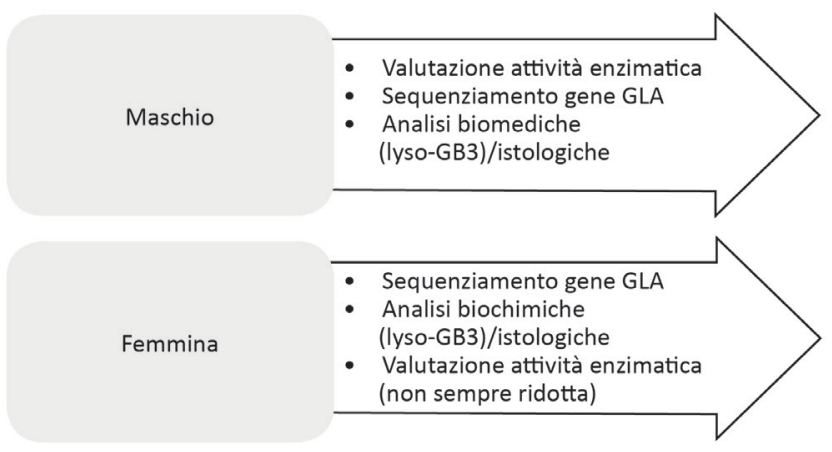

Fig. 1 - L'iter diagnostico della malattia di Fabry.

di eventi cardiovascolari e renali maggiori (7-9). La ERT può quindi ridurre la progressione della malattia soprattutto se iniziata precocemente e incide anche sulla qualità della vita $\mathrm{e}$ sulla deposizione di Gb3 nei vari organi (10).

Recentemente è stato dimostrato come alcuni pazienti possano beneficiare anche della terapia a base di migalastat (11); questo chaperon, somministrato per via orale, è in grado di stabilizzare specifiche forme mutanti di alfa-galattosidasi A. La terapia con migalastat è in grado di stabilizzare l'enzima ed è indicata solo in caso di specifiche mutazioni (cosiddette amenable o suscettibili) che non determinano alterazioni a livello del sito attivo dell'enzima stesso.

\section{Il ruolo del nefrologo nella diagnosi di malattia di Fabry}

La nefropatia in corso di malattia di Anderson-Fabry nella variante classica è in genere caratterizzata dall'insorgenza di microalbuminuria che esordisce già in età giovanile, a cui fa seguito lo sviluppo di un danno renale cronico già nella terza-quarta decade di vita (più comune nei soggetti di sesso maschile). Nei soggetti di sesso femminile sono descritti quadri clinici estremamente variabili per cui potremo osservare pazienti completamente asintomatici oppure con interessamento renale indistinguibile da quello dei soggetti di sesso maschile. Alcuni autori hanno descritto anche una maggiore incidenza di cisti parapieliche rispetto alla popolazione generale (12), isostenuria e alterazione della funzione tubulare. La patogenesi della nefropatia nel paziente affetto da malattia di Fabry è legata a un processo di accumulo di glicosfingolipidi a livello lisosomiale ed extralisosomiale. L'accumulo di substrato provoca un danno lisosomiale con deregolazione dei meccanismi di autofagia, alterazioni nel metabolismo mitocondriale, apoptosi con conseguente proteinuria e progressione del danno renale. I depositi di Gb3 e lyso-Gb3 possono essere osservati a livello dei podociti e delle cellule endoteliali (fasi precoci) con conseguente danno microvascolare (13), delle cellule mesangiali e del tubulo distale (fasi tardive). Nelle fasi tardive della malattia si assiste allo sviluppo di fibrosi interstiziale, atrofia tubulare e glomerulosclerosi (danno irreversibile). Recentemente alcuni autori hanno proposto come possibile marker precoce della nefropatia la presenza di una fusione dei pedicelli dei podociti e il conseguente distacco dalla membrana basale con comparsa di podocituria. Quest'ultimo fenomeno sembra precedere lo sviluppo della proteinuria e il conseguente declino della funzione renale (14). Le alterazioni istopatologiche comunemente osservabili alla biopsia renale sono in genere quelle di un caratteristico aspetto "a nido d'ape" dovuto alla presenza di vacuolizzazione a livello dei podociti, fenomeno dovuto all'estrazione dei depositi di sfingolipidi che avviene nell'allestimento del preparato. La microscopia elettronica consente di evidenziare, a livello delle cellule endoteliali, i caratteristici depositi lamellari "zebra bodies". Le principali alterazioni istopatologiche che possono essere riscontrate alla biopsia renale sono la sclerosi segmentaria o globale, la fibrosi interstiziale, la sclerosi arteriolare, i vacuoli podocitari e inclusi tubulo-arteriolari (15). Clinicamente le prime fasi della nefropatia sono caratterizzate da uno stato di iperfiltrazione glomerulare con caratteristiche simili a quelle osservate nella nefropatia diabetica a cui può essere associata la presenza di microalbuminuria (13). Nelle fasi più avanzate della malattia lo sviluppo di fibrosi, conseguenza del danno ischemico secondario all'accumulo di glicosfingolipidi, rappresenta un evento irreversibile che determina la progressione della nefropatia verso l'insufficienza renale cronica condizionando notevolmente l'efficacia della ERT (16). In considerazione della iperfiltrazione glomerulare i pazienti affetti da nefropatia in corso di malattia di Fabry trovano un beneficio importante dalla assunzione di $\mathrm{ACE}$-inibitori o $\mathrm{ARBs}$ che risulta ancora più importante quando associato con la ERT. L'efficacia della terapia con tali farmaci non è solo legata all'effetto antiproteinurico della terapia ma anche all'inibizione dell'effetto profibrotico legato ai componenti del sistema renina-angiotensina-aldosterone (SRAA). Vari studi dimostrano come la combinazione della ERT con gli inibitori del SRAA sia in grado non solo di ridurre la proteinuria ma anche la progressione del danno renale cronico (17). Complessivamente l'obiettivo della terapia è quello di rallentare la progressione del danno renale e cercare di ridurre la proteinuria almeno a valori inferiori a $1 \mathrm{~g} / 24 \mathrm{~h}$ (target $0,5 \mathrm{~g} / 24 \mathrm{~h}$ ) anche nel caso in cui i valori di pressione arteriosa risultino inferiori a 130/80 $\mathrm{mmHg}$, riducendo la progressione del danno renale cronico (7). In letteratura viene anche riportato un ulteriore beneficio in termini di riduzione della proteinuria associando analoghi della vitamina $\mathrm{D}$ (paracalcitolo) anche in assenza di iperparatiroidismo secondario (18). 


\section{Il ruolo del nefrologo nella gestione multidisciplinare del paziente}

Date queste premesse appare evidente come il nefrologo abbia un ruolo essenziale nella diagnosi della malattia e quindi nell'identificazione di quei pazienti che necessitano l'avviamento di una terapia specifica. Tale considerazione tuttavia non rappresenta un elemento di merito dello specialista e un fatto positivo per il paziente poiché spesso la diagnosi viene posta tardivamente. Una strategia di notevole impatto potrebbe essere quella di promuovere un programma di screening neonatale in grado di consentire una precoce identificazione di pazienti e successivamente di eventuali membri familiari asintomatici e non diagnosticati, come è avvenuto in alcuni studi pilota. Nell'ambito degli screening, considerando l'alta frequenza di soggetti nefropatici senza una causa nota, è di grande aiuto lo screening nella popolazione di pazienti con ESRD che ha riportato una prevalenza della malattia compresa tra lo 0,1 e lo 0,3\% (19).

Una volta posta la diagnosi di malattia di Fabry i pazienti potranno essere presi in carico da una équipe multidisciplinare composta da cardiologo, nefrologo, neurologo, genetista, oculista, dermatologo, otorinolaringoiatra, specialista in malattie metaboliche, gastroenterologo, che avrà lo scopo di facilitare il complesso iter necessario per il corretto inquadramento dell'interessamento multiorgano, determinare il pedigree familiare e stabilire la cadenza del follow-up. Né si deve dimenticare il ruolo non marginale di una valutazione psicologica con l'ausilio di scale codificate per caratterizzare e monitorare la qualità della vita dei pazienti (Raw score, DS3, MSSI) e ulteriori valutazioni specialistiche in casi particolari di interessamento polmonare e/o a carico di altri organi e apparati. All'interno di questo gruppo, indubbiamente il nefrologo riveste un ruolo centrale soprattutto perché la malattia tende a progredire interessando organi maggiori come il SNC, il cuore e appunto il rene portando questi ultimi, spesso, a una insufficienza funzionale che nel caso del rene, può necessitare della dialisi o del trapianto. Quindi il compito di svolgere principalmente un'opera preventiva sulla progressione della malattia deve essere del nefrologo.

Il nefrologo ha il compito di impostare un work-up completo della malattia che deve comprendere la ricerca di albuminuria, la valutazione del sedimento urinario, il controllo degli indici di funzione renale, il dosaggio del lyso-Gb3, una ecografia addome. I pazienti devono essere stratificati in base alla funzione renale, l'entità della proteinuria (albuminuria e proteinuria nelle 24 ore o rapporto albuminuria/creatininuria), valori di pressione arteriosa, livelli di lyso-Gb3, tipo di mutazione genetica riscontrata, presenza di eventuali anticorpi anti-ERT, in modo tale da intervenire per stabilizzare la nefropatia e rallentare la perdita del filtrato glomerulare. La valutazione nefrologica avviene a intervalli variabili (ogni 3-12 mesi) a seconda dello stadio di malattia renale cronica (K/DOQI) e in base all'entità della proteinuria (20). Un altro

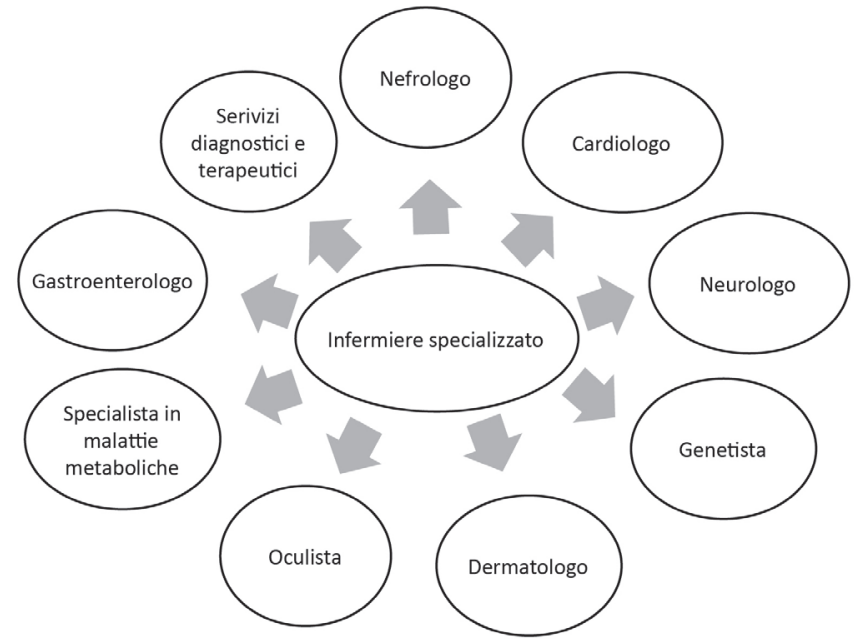

Fig. 2 - L'équipe multidisciplinare nella gestione della malattia di Fabry.

elemento di primaria importanza che caratterizza la competenza nefrologica per la cura del paziente con malattia di Fabry è l'indicazione e l'esecuzione della biopsia renale che insieme al controllo periodico degli indici di funzione renale, della proteinuria e del filtrato glomerulare, rappresenta un cardine sia per l'inizio della terapia enzimatica sostitutiva sia per valutare la risposta alla terapia. L'esecuzione della biopsia renale ha lo scopo di identificare precocemente l'interessamento d'organo della malattia, escludere coesistenza di altre patologie, valutare l'estensione della deposizione di GL-3. Tale indagine diagnostica può anche rappresentare un elemento utile alla valutazione della risposta alla ERT e deve essere eseguita soprattutto nei pazienti che presentano albuminuria e/o proteinuria, nei soggetti di sesso maschile con presentazione atipica e nelle femmine con prima manifestazione della patologia (soprattutto nei casi in cui si sospetti la coesistenza di un'altra patologia glomerulare e nei casi di progressione rapida del danno renale). La dimostrazione istologica di un interessamento renale che può precedere la manifestazione clinica di malattia consente di iniziare la ERT precocemente e prevenire l'accumulo di glicosfingolipidi nel parenchima renale consentendo di rallentare la progressione del danno. II nefrologo è anche coinvolto nella gestione della ERT nel paziente trapiantato di rene e nel paziente in trattamento dialitico in cui l'indicazione alla terapia è legata al controllo della progressione del danno extrarenale.

Data la complessità dell'iter diagnostico fin qui descritto all'interno dell'équipe multidisciplinare gioca un ruolo di primo piano l'infermiere coordinatore (Fig. 2). Tale figura professionale ha la funzione di raccordo tra gli specialisti in modo tale da garantire una programmazione delle visite specialistiche e degli esami strumentali a cui il paziente dovrà sottoporsi con possibilità anche di accorpamento, riducendo il disagio di accessi multipli. Tale approccio risulta 
particolarmente importante per quanto riguarda la gestione delle famiglie da studiare residenti in luoghi lontani dal Centro specialistico di riferimento. Infine l'équipe multidisciplinare ha il compito di svolgere periodici incontri per discutere le tematiche cliniche dei singoli casi come per esempio stabilire l'inizio della ERT, la sua cessazione, l'eventuale passaggio da una terapia all'altra o l'arruolamento dei pazienti in protocolli sperimentali. Data la variabilità dell'interessamento d'organo della malattia l'équipe multidisciplinare potrà rilasciare una documentazione in cui si riassume l'entità dell'interessamento a carico dei singoli apparati e indicare le motivazioni di una eventuale scelta terapeutica e il tipo di follow-up. La funzione dell'équipe multidisciplinare risulterà anche essenziale nell'aggiornamento scientifico, data la notevole evoluzione delle conoscenze sulla malattia di Fabry avvenute in particolare nell'ultimo decennio.

\section{Disclosures}

Financial support: No financial support was received for this submission.

Conflict of interest: The authors have no conflict of interest.

\section{Bibliografia}

1. Feriozzi S. [Glomerular lipidosis]. Malattie Metaboliche e Rene. G Ital Nefrol. 2016;33(S68):74-77.

2. Mehta A, Ricci R, Widmer $U$, et al. Fabry disease defined: baseline clinical manifestations of 366 patients in the Fabry Outcome Survey. Eur J Clin Invest. 2004;34(3):236-42.

3. Hollak CE, Weinreb NJ. The attenuated/late onset lysosomal storage disorders: Therapeutic goals and indications for enzyme replacement treatment in Gaucher and Fabry disease. Best Pract Res Clin Endocrinol Metab. 2015;29(2):205-18.

4. Eng CM, Fletcher J, Wilcox WR, et al. Fabry disease: baseline medical characteristics of a cohort of 1765 males and females in the Fabry Registry. J Inherit Metab Dis. 2007; 30(2): 184-92.

5. Mignani R, Gallieni M, Feriozzi S, Pisani A, Marziliano N, Morrone A. [The nephropathy in the Anderson-Fabry disease: new recommendations for the diagnosis, the follow-up and the therapy]. G Ital Nefrol. 2015;32(4).

6. Schiffmann R, Waldek S, Benigni A, Auray-Blais C. Biomarkers of Fabry disease nephropathy. Clin J Am Soc Nephrol. 2010;5(2): 360-4.
7. Germain DP, Charrow J, Desnick RJ, et al. Ten-year outcome of enzyme replacement therapy with agalsidase beta in patients with Fabry disease. J Med Genet. 2015;52(5):353-8.

8. Schiffmann R, Swift C, Wang X, Blankenship D, Ries M. A prospective 10-year study of individualized, intensified enzyme replacement therapy in advanced Fabry disease. J Inherit Metab Dis. 2015;38(6):1129-36.

9. Beck M, Hughes D, Kampmann C, et al. Long-term effectiveness of agalsidase alfa enzyme replacement in Fabry disease: A Fabry Outcome Survey analysis. Mol Genet Metab Rep. 2015;3:21-7.

10. El Dib R, Gomaa H, Carvalho RP, et al. Enzyme replacement therapy for Anderson-Fabry disease. Cochrane Database Syst Rev. 2016;7:CD006663.

11. Germain DP, Hughes DA, Nicholls K, et al. Treatment of Fabry's Disease with the Pharmacologic Chaperone Migalastat. N Engl J Med. 2016;375(6):545-55.

12. Sayer JA, Haslam P, Brennan P. Parapelvic cysts leading to a diagnosis of Fabry disease. Kidney Int. 2008;74(10):1366.

13. Torra R, Ortiz A. Fabry disease: the many faces of a single disorder. Clin Kidney J. 2012;5:379-82.

14. Trimarchi H, Canzonieri R, Schiel A, et al. Podocyturia is significantly elevated in untreated vs treated Fabry adult patients. J Nephrol. 2016;29(6):791-7.

15. Fogo $A B$, Bostad L, Svarstad E, et al. Scoring system for renal pathology in Fabry disease: report of the International Study Group of Fabry Nephropathy (ISGFN). Nephrol Dial Transplant. 2010;25(7):2168-77.

16. Weidemann F, Sanchez-Niño MD, Politei J, et al. Fibrosis: a key feature of Fabry disease with potential therapeutic implications. Orphanet J Rare Dis. 2013 Aug 6;8:116.

17. Warnock DG, Thomas CP, Vujkovac B, et al. Antiproteinuric therapy and Fabry nephropathy: factors associated with preserved kidney function during agalsidase-beta therapy. J Med Genet. 2015;52(12):860-6.

18. Pisani A, Sabbatini M, Duro G, Colomba P, Riccio E. Antiproteinuric effect of add-on paricalcitol in Fabry disease patients: a prospective observational study. Nephrol Dial Transplant. 2015;30(4):661-6.

19. Linthorst GE, Bouwman MG, Wijburg FA, Aerts JM, Poorthuis BJ, Hollak CE. Screening for Fabry disease in high-risk populations: a systematic review. J Med Genet. 2010;47(4):217-22.

20. Terryn W, Cochat P, Froissart R, et al. Fabry nephropathy: indications for screening and guidance for diagnosis and treatment by the European Renal Best Practice. Nephrol Dial Transplant. 2013;28(3):505-17. 